\title{
Role of the disk environment in the gamma-ray emission from the binary system PSR B1259-63/LS 2883
}

\author{
lurii Sushch* \\ Centre for Space Research, North-West University, Potchefstroom 2520, South Africa \\ Astronomical Observatory of Ivan Franko National University of L'viv, vul. Kyryla i Methodia, \\ 8, L'viv 79005, Ukraine \\ E-mail: iurii.sushchenwu.ac.za
}

\section{Markus Böttcher ${ }^{\dagger}$}

Centre for Space Research, North-West University, Potchefstroom 2520, South Africa

\begin{abstract}
PSR B1259-63/LS 2883 is a very high energy (VHE; E > $100 \mathrm{GeV}$ ) gamma-ray emitting binary consisting of a $48 \mathrm{~ms}$ pulsar orbitting around a Be star with a period of 3.4 years. The Be star features a circumstellar disk which is inclined with respect to the orbit in such a way that the pulsar crosses it twice every orbit. The circumstellar disk provides an additional field of target photons which may contribute to inverse Compton scattering and gamma-gamma absorption, leaving a characteristic imprint in the observed spectrum and light curve of the high energy emission. We study the signatures of Compton-supported, VHE gamma-ray induced pair cascades in the circumstellar disc of the Be star and their possible contribution to the GeV flux. We also study a possible impact of the gamma-gamma absorption in the disk on the observed $\mathrm{TeV}$ light curve. We show that the cumulative absorption of VHE gamma-rays in stellar and disk photon fields can explain the modulation of the flux at the periastron passage.
\end{abstract}

The 34th International Cosmic Ray Conference,

30 July- 6 August, 2015

The Hague, The Netherlands

*Speaker.

$\dagger$ The work of M.B. is supported by the South African Research Chair Initiative (SARChI) of the South African National Research Foundation and the Department of Science and Technology. Disclaimer: Any opinion, finding and conclusion or recommendation expressed in this material is that of the authors and the NRF does not accept any liability in this regard. 


\section{Introduction}

PSR B1259-63/LS 2883 is a member of the small class of very high energy (VHE; $E>100$ $\mathrm{GeV}) \gamma$-ray binaries which comprises only five known objects. PSR B1259-63/LS 2883 is unique for being the only $\gamma$-ray binary for which the compact object is clearly identified as a pulsar. This pulsar with a spin period of $\simeq 48 \mathrm{~ms}$ and a spin-down luminosity of $\simeq 8 \times 10^{35} \mathrm{erg} \mathrm{s}^{-1}$ is moving in a highly eccentric $(e=0.87)$ orbit around a massive Be star with a period of $P_{\text {orb }}=3.4$ years $(1237$ days) [1, 2]. The companion star LS 2883 has a luminosity of $L_{*}=2.3 \times 10^{38} \mathrm{erg} \mathrm{s}^{-1}$. Because of the fast rotation of the star, it has an oblate shape with an equatorial radius of $R_{\mathrm{eq}}=9.7 R_{\odot}$ and a polar radius of $R_{\text {pole }}=8.1 R_{\odot}$, which in turn leads to a strong gradient of the surface temperature from $T_{\text {eq }} \simeq 27,500 \mathrm{~K}$ at the equator to $T_{\text {pole }} \simeq 34,000 \mathrm{~K}$ at the poles [3].

The Be star features an equatorial circumstellar disk, which is believed to be inclined with respect to the pulsar's orbit (see e.g. [3]), so that the pulsar crosses the disk twice each orbit. The circumstellar disk of a Be star is a decretion disk with an enhanced stellar outflow formed around the star. As shown in optical interferometry observations, these disks are symmetrical with respect to the star's rotation axis (see e.g. [4]). Circumstellar disks generate excess infrared (IR) emission produced through free-free and free-bound radiation, providing an additional IR photon field to the blackbody flux from the optical star.

The dense medium of the disk is believed to play an essential role in the resulting emission from the system. The position of the disk can be localized based on the disappearance of the pulsed radio emission from the pulsar. The observed radio emission far from periastron consists only of the pulsed component [5,6], but closer to periastron, at about $t_{\mathrm{p}}-100 \mathrm{~d}\left(t_{\mathrm{p}}\right.$ is the time of periastron), the intensity of the pulsed emission starts to decrease and completely disappears at about $t_{\mathrm{p}}-20 \mathrm{~d}$. It then re-appears at around $t_{\mathrm{p}}+15 \mathrm{~d}$. This eclipse of the pulsed emission is believed to be caused by the circumstellar disk. It is accompanied by an increase of the transient unpulsed radio flux beginning at $\sim t_{\mathrm{p}}-30 \mathrm{~d}$ and reaching its maximum at $\sim t_{\mathrm{p}}-10 \mathrm{~d}$. This is followed by a decrease around the periastron passage and a second peak at about $t_{\mathrm{p}}+20 \mathrm{~d}[5,6,7]$. A similar behavior is observed also for the unpulsed X-ray emission. Close to the periastron passage the X-ray emission features two peaks at around 20 days before and after periastron with flux levels $10-20$ times higher than during apastron, and a decrease of the emission at the time of the periastron passage itself. The X-ray data is very similar from orbit to orbit repeating the shape of the light curve very well ([7] and references therein). These peaks might be connected to the crossing of the disk environment.

At $\mathrm{TeV}$ energies the source was observed by H.E.S.S. around four periastron passages in 2004 [8], 2007 [9], 2010 [10], and 2104 (results not available yet). The TeV emission from the source shows a variable behavior around the periastron passage. Although the exposure of the source is not sufficient to draw firm conclusions, the combined light curves from all three observing campaigns show a hint of two asymmetrical peaks before and after periastron, which roughly coincide in time with the peaks of the emission in the radio and X-ray bands, and a decrease of the flux at periastron. The shape of the light curve might be a result of the interaction of the pulsar with the circumstellar disk.

First observations at GeV energies conducted by Fermi-LAT took place around the 2010 periastron passage revealing quite unexpected results [11, 12]. Fermi-LAT detected a low flux from 
the source close to periastron with a subsequent dissapearance of the source after periastron followed by a sudden flare (with $\sim 10$ times the pre-periastron flux level) 30 days after periastron. This flare lasted for about 7 weeks without any obvious counterparts at other energy bands. The flare is shifted in time with respect to the post-periastron peak at other energy bands. The nature of the flare is still not understood, but several possible explanations have been discussed in the literature $[13,14,15,16,16,17]$. New observations around the 2014 periastron passage revealed the $\mathrm{GeV}$ flare at the similar orbital phase establishing the repetitive behaviour of this phenomenon [18]. However, new observations did not show any significant emission close to periastron [18].

The circumstellar disk of the companion star plays a crucial role in the variability of the $\gamma$-ray emission from the system. The dense disk photon field should significantly contribute to the target photons for inverse Compton scattering from the source, enhancing the observed $\mathrm{TeV}$ emission. The abrupt change of density at the pulsar's entrance and escape from the disk should also cause a change of the shape of the shock between the pulsar wind and stellar environment, which might be the reason of such spectacular events as the GeV flare. The high density of seed photons should also increase the opacity for $\gamma \gamma$-absorption, followed by electron-positron pair production, which in turn might scatter again the disk photon field, generating secondary $\gamma$-rays. This cascade process may cause the re-emission of the TeV IC flux at lower $\mathrm{GeV}$ energies. Moreover, because of the deflection of electrons and positrons in the magnetic field, secondary $\gamma$-rays can be re-emitted into randomized directions. Therefore, even if the primary VHE photon was emitted into the direction opposite to the line of sight to the observer, it can still contribute to the observable flux at lower $\mathrm{GeV}$ energies through the cascade emission. The pair cascade emission in binary systems caused by the interaction of the primary very high energy photons with the stellar photons was studied in detail for several cases (see e.g. [19, 20] and references therein). These studies showed that, although environments of the binary systems fulfill all the requirements for effective pair cascading, the resulting spectrum is in conflict with the $\mathrm{GeV}$ data observed by Fermi-LAT, since the expected cascade emission peaks in the Fermi-LAT energy band with very constraining upper limits. The $\gamma \gamma$-absorption of the $\mathrm{TeV} \gamma$-rays by stellar photons was also suggested as a possible explanation of the variability of the $\mathrm{TeV}$ flux across the orbit [21]. The idea is that at periastron, when the pulsar is at the shortest distance to the star, the absorption should be the most effective, which would provide the decrease of the flux. However, [21] showed that absorption only cannot explain the TeV light curve.

\section{Cascade Emission in the Circumstellar Disk}

We used the cascade Monte Carlo code of $[22,23]$ to calculate the angle-dependent cascade spectra for a variety of different input parameter sets within a parameter space motivated by the known properties of the Be star and its circumstellar disk in the PSR B1259-63/LS 2883 binary system. In these calculations we assume that the pulsar is a point-like source emitting isotropically in all directions. This assumption is reasonable if the wind termination shock at which leptons injected by the pulsar are efficiently accelerated and isotropized is close to the pulsar and the density of target seed photons is high enough to lead to IC scattering in the direct vicinity of the shock, which is the case for PSR B1259-63/LS 2883 [24]. We approximate the spectrum of the VHE $\gamma$-rays as generated by IC scattering by electrons as a power law distribution with an 
exponential cut-off. In our simulations we consider a mono-directional beam of photons to isolate all the geometrical effects. The source would emit the same beam of photons in every direction, and in the case of efficient cascading even those photons emitted in the opposite direction from the observer can contribute to the resulting observable spectrum. The orientation of the magnetic field would be different for primary photons emitted in different directions. The disk is approximated by a grey-body with the energy density $u_{\mathrm{d}}$ and temperature $T_{\mathrm{d}}=0.6 T_{*}[25,26,27]$ and the shape of the disk is approximated by a cuboid whose side lengths are chosen in a way to correspond to the real size of the disk. All the details on model assumptions and their justification can be found in [24].

The dependence of the cascade emission on the viewing angle, magnetic field strength and orientation, energy density in the disk, and source location within the disk were discussed in detail in [24]. It was also shown that the GeV flare is very unlikely to be genereated by the cascade emission. However, cascade emission might still contribute to the faint $\mathrm{GeV}$ emission observed close to periastron during the periastron passage in 2010. The spectrum presented by [12] is consistent with a substantial contribution from cascade emission. The energy of the peak in the spectrum of the cascade $\gamma$-rays emitted in the forward direction only slightly depends on the energy density and magnetic field [24] and is in the energy range of $15-30 \mathrm{GeV}$. Figure 1 shows the comparison of the $\mathrm{GeV}-\mathrm{TeV}$ data with the simulation results of the cascade Compton radiation from the primary photon beam directed towards the observer emitted in the forward direction into a cone with an opening angle $11^{\circ}(0.98 \leq \mu \leq 1)$. This corresponds to the cascade emission radiated in the direction to the observer from the primary $\gamma$-rays emitted within the cone of the same opening angle assuming that the magnetic field affects the cascades initiated by every primary photon identically. This assumption is valid for sufficiently small values of the opening angle, such that the orientation of the magnetic field with respect to the direction of the primary photon does not change much and its influence on the shape of the cascade emission spectrum is negligible. The simulation was performed for $u_{\mathrm{d}}=20 \mathrm{erg} / \mathrm{cm}^{3}$, magnetic field $B=0.1 \mathrm{G}$ with $\beta=B_{\mathrm{x}} / B_{\mathrm{y}}=10$ (B lies in the $x y$-plane). We decided to chose a magnetic-field direction closely aligned with the primary photon beam (and thus, the direction to the observer). In this way, the magnetic field has very little influence on the cascade development and on the resulting Compton emission. Otherwise, the cascade radiation emitted in other directions can become higher than the forward emission and a cone with a bigger opening angle should be considered, which in turn requires an accurate calculation of the magnetic field orientation for every primary photon.

However, the spectrum of the $\mathrm{GeV}$ emission during the "low-flux" period derived by an independent group features a completely different spectral shape [11]. It reveals a significant flux at $0.1-1 \mathrm{GeV}$ with no emission above $1 \mathrm{GeV}$. In this case the spectrum rejects the possibility of a significant cascade contribution, because even a very small level of absorption of the $\mathrm{TeV} \gamma$-rays and re-emission at lower energies would violate the Fermi-LAT upper limit in $1-100 \mathrm{GeV}$ energy band. The Fermi-LAT upper limit in this case constrains the energy density in the disk to $u_{\mathrm{d}} \lesssim 8$ $\mathrm{erg} / \mathrm{cm}^{3}$ [24]. Unfortunately, no significant emission close to periastron was detected during the 2014 periastron passage [18], thus, the spectral discrepancy remains unresolved.

It should be noted though that the simulations presented above are performed considering only the disk radiation field and neglecting the stellar radiation field. Therefore, the comparison of the model with the "periastron" emission presented on Fig. 1 should be treated only as a hint that the 


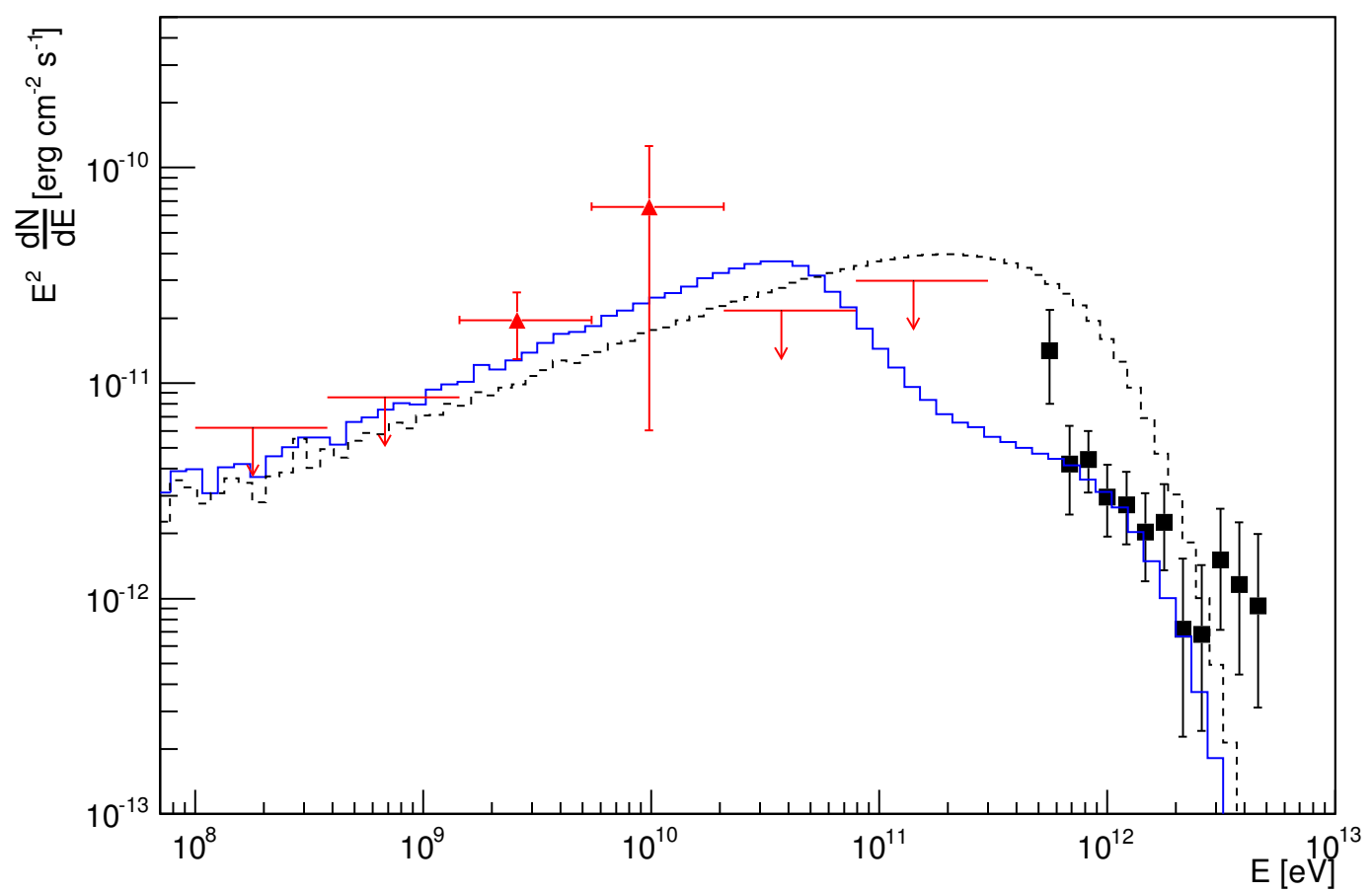

Figure 1: Comparison with the data. The black dashed line represents the intrinsic $\gamma$-ray spectrum. The blue solid line represents the spectrum of the forward emission $(0.98 \leq \mu \leq 1)$ modified by gamma-gamma absorption and cascading. Assumed parameters: $B=0.1 \mathrm{G}, \beta=10, u_{\mathrm{d}}=20 \mathrm{erg} / \mathrm{cm}^{3}$. Black squares represent the 2011 H.E.S.S. data [10] and red triangles show the $\mathrm{GeV}$ data close to the 2010 periastron passage as reported by [12].

$\mathrm{GeV}$ emission may be explained by the cascade radiation (even if we consider only disk photons). This implies that taking into account stellar photons might probably provide a better fit of the data and decrease the estimate (upper limit) of the disk energy density. A detailed study of the cascade development in the system which would properly account for all relevant effects is in progress.

\section{Impact of the $\gamma \gamma$-Absorption on the Observed TeV Light Curve}

The absorption of the $\gamma$-ray emission caused by the interaction with the disk radiation field might have a significant impact at the observed $\mathrm{TeV}$ light curve. The resulting integrated flux above $380 \mathrm{GeV}$ after absorption caused by the stellar radiation field was calculated in [21]. It was shown that the absorption due to the stellar radiation plays only a minor role in the observed variability. However, the disk radiation was not considered in those calculations. Using the estimate of the resulting integrated flux after the absorption on stellar photons only, $F_{\mathrm{s}}$, calculated in [21] we calculated the resulting flux after the total absorption due to both stellar and disk radiation fields as

$$
F(E>380 \mathrm{GeV}) \simeq F_{\mathrm{s}}(E>380 \mathrm{GeV}) e^{-\bar{\tau}_{\gamma \gamma}}=F_{\mathrm{S}}(E>380 \mathrm{GeV}) \frac{\int_{\varepsilon_{\gamma, \min }}^{\varepsilon_{\gamma, \max }} e^{-\tau_{\gamma \gamma \mathrm{d}}\left(\varepsilon_{\gamma}\right)} d \varepsilon_{\gamma}}{\varepsilon_{\gamma, \max }-\varepsilon_{\gamma, \min }},
$$


assuming the optical depth of the disk radiation field $\tau_{\gamma \gamma, \mathrm{d}}(\varepsilon)$ only weekly depends on energy $\left(\varepsilon_{\gamma}=E_{\gamma} /\left(m_{\mathrm{e}} c^{2}\right)\right.$ is the normalised $\gamma$-ray energy) in the energy range $E_{\gamma, \min }=380 \mathrm{GeV}$ and $E_{\gamma, \max }=$ $10 \mathrm{TeV}$ and can be replaced by a constant averaged value $\bar{\tau}_{\gamma \gamma}$. For these calculations we used the same orbital parameters as used in [21]: orbital period $P_{\text {orb }}=1236.7$, eccentricity $e=0.87$, periastron longitude $\omega=138.7^{\circ}$, and inclination angle $i=35^{\circ}$. The disk inclination angle is assumed to be $i_{\mathrm{d}}=10^{\circ}$. The disk is assumed to be perpendicular to the major axis of the orbit with a constant width of $10^{12} \mathrm{~cm}$ (corresponds to $1^{\circ}$ half-opening angle at a distance of $45 R_{*}$ ) and constant energy density of $8 \mathrm{erg} \mathrm{cm}^{-3}$ (the highest energy density for which the Fermi-LAT upper limits are not violated, see previous subsection). The expected light curve after the total absorption assuming a constant initial $\gamma$-ray flux is shown in Fig. 2 by the dashed line. The solid line represents the flux after absorption taking into account only stellar photons as reported by [21]. However, the assumption of the constant optical depth underestimates the effect of the $\gamma \gamma$-absorption in the disk as the optical depth $\tau_{\gamma \gamma, \mathrm{d}}(\varepsilon)$ peaks at $\sim 300 \mathrm{GeV}$. The resulting light curve after absorption in the disk only taking into account dependence of the optical depth on energy is shown by the dotted line in Fig. 2.

It can be seen that the circumstellar disk might play a crucial role in the flux variability around the periastron passage and that the observed flux variability can be explained by the absorption. The time shift of the observed preperiastron peak with respect to the modelled light curve might be explained by the rotation of the disk normal with respect to the major axis. The assumption of the constant width of the disk results in an overestimation of the absorption close to periastron, since the disk should be thinner close to the star. However, the energy density in the disk is expected to be highest close to the star. The combined effect might result in a shallower minimum near periastron than predicted by our calculations. The light curve has been modelled under the assumption that the primary $\gamma$-ray flux is constant. This is a valid approximation only in the case when IC scattering occurs in the saturation regime which is believed to be true around periastron. Farther from periastron the primary flux is expected to decrease. In the case when IC scattering is not in the saturation regime, local maxima are expected at the disk crossings and at the periastron. This would completely change the expected light curve. With the current quality of the observed TeV light curve it is difficult to draw any final conclusions. New observations conducted in 2014 might bring some clarity and give answers to some of these open questions.

A critical test of the absorption model could be derived from the study of the spectral variability of the $\mathrm{TeV}$ emission. The TeV spectrum is expected to soften when the pulsar is moving through the disk for the first time, then the spectral shape should remain almost unchanged with a slight variability due to the absorption in the stellar radiation field until the pulsar moves out of the disk after periastron. After that the spectrum should harden again. Unfortunately, the VHE $\gamma$-ray observations during previous periastron passages did not provide enough statistics to draw firm conclusions about the spectral variability. Although there are some indications of spectral changes both during the 2004 [8] and the 2007 [9] periastron passages, these changes are not significant. However, if the spectral variability is real on both occasions, it suggests a hardening of the spectrum towards periastron, which is in contradiction with the effect we expect in the case of effective cascading.

The study of the $\gamma \gamma$-absorption in the disk which would take into account a proper geometry of the disk and a realistic distribution of IR photons within the disk corresponding to the observed 


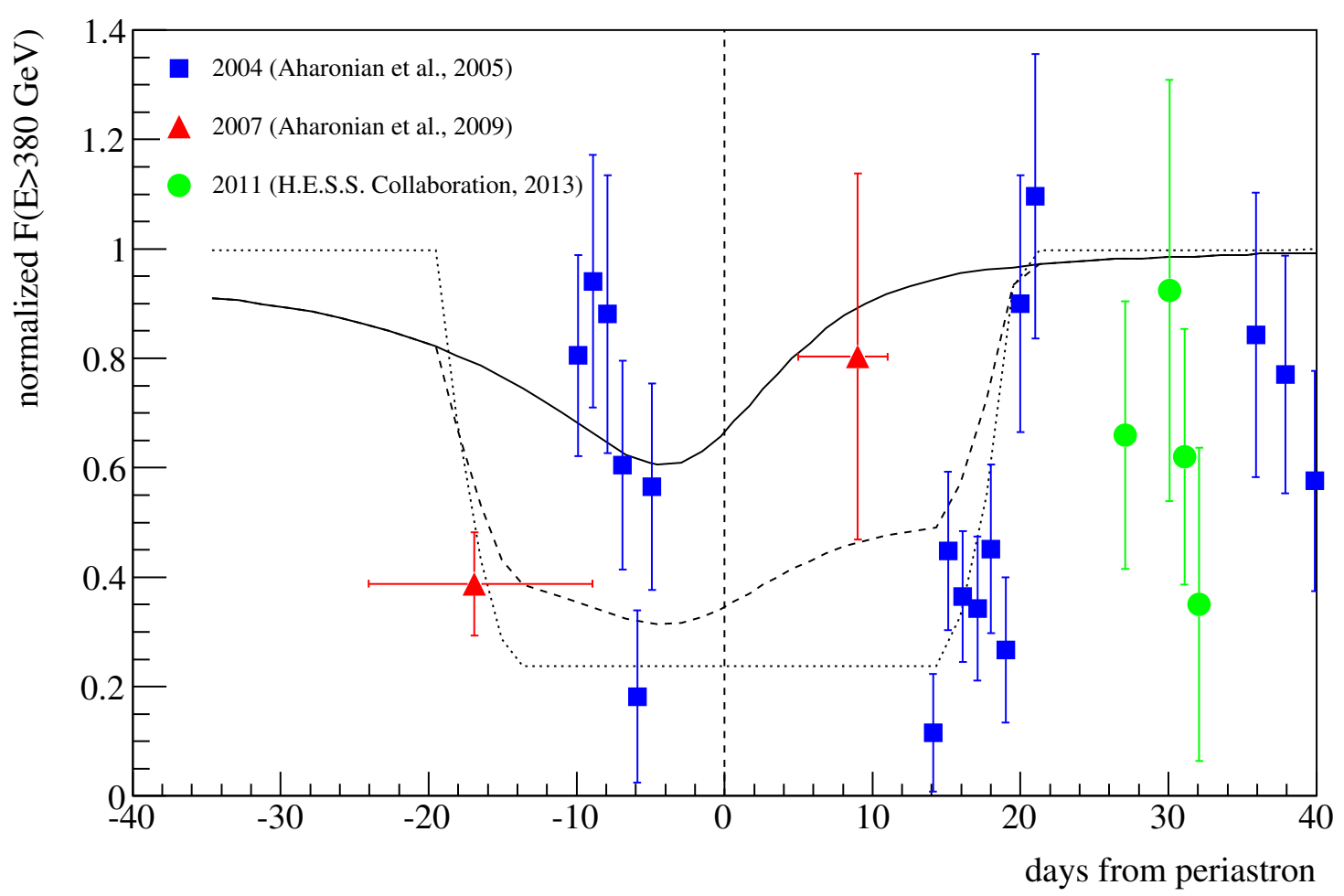

Figure 2: VHE $\gamma$-ray flux modulation caused by $\gamma \gamma$-absorption in PSR B1259-63/LS 2883. The solid line represents the normalized integrated flux above $380 \mathrm{GeV}$ after absorption caused by the stellar radiation field as calculated in [21]. The dashed line shows the same flux taking into account also the absorption caused by the disk radiation field assuming the $\gamma \gamma$ optical depth is constant above $380 \mathrm{GeV}$. The dotted line represents the normalized integrated flux above $380 \mathrm{GeV}$ after absorption caused by the circumstellar disk only taking into account the dependancy of the $\gamma \gamma$ optical depth on energy. See the main text for details on the geometry and other parameters. The data points represent the combined H.E.S.S. light curve from three observed periastron passages as reported in [10]. The integrated flux above $1 \mathrm{TeV}$ is extrapolated down to $380 \mathrm{GeV}$ using a photon index of 2.8. The same flux normalisation factor as in [21] of $10^{-11} \mathrm{~cm}^{-2} \mathrm{~s}^{-1}$ is used.

IR emission from the star is currently in progress.

\section{References}

[1] S. Johnston, et al., A high-frequency survey of the southern Galactic plane for pulsars, MNRAS, 255 (Apr., 1992) 401-411.

[2] S. Johnston, et al., PSR 1259-63 - A binary radio pulsar with a Be star companion, ApJ, 387 (Mar., 1992) L37-L41.

[3] I. Negueruela, et al., Astrophysical Parameters of LS 2883 and Implications for the PSR B1259-63 Gamma-ray Binary, ApJ, 732 (May, 2011) L11, [arXiv:1103.4636].

[4] A. Quirrenbach, et al., Maximum-entropy maps of the Be shell star zeta Tauri from optical long-baseline interferometry, A\&A, 283 (Mar., 1994) L13-L16.

[5] S. Johnston, et al., Transient radio emission from the PSR B1259-63 system near periastron, MNRAS, 302 (Jan., 1999) 277-287. 
[6] S. Johnston, et al., Radio observations of PSR B1259-63 through the 2004 periastron passage, MNRAS, 358 (Apr., 2005) 1069-1075, [astro-ph/ 0501660 ].

[7] M. Chernyakova, et al., Multiwavelength observations of the binary system PSR B1259-63/LS 2883 around the 2010-2011 periastron passage, MNRAS, 439 (Mar., 2014) 432-445, [arXiv:1401.1386].

[8] F. Aharonian, et al., Discovery of the binary pulsar PSR B1259-63 in very-high-energy gamma rays around periastron with HESS, A\&A, 442 (Oct., 2005) 1-10, [a stro-ph/ 0506280 ].

[9] F. Aharonian, et al., Very high energy $\gamma$-ray observations of the binary PSR B1259-63/SS2883 around the 2007 Periastron, A\&A, 507 (Nov., 2009) 389-396.

[10] H.E.S.S. Collaboration, A. Abramowski, et al., H.E.S.S. observations of the binary system PSR B1259-63/LS 2883 around the 2010/2011 periastron passage, A\&A, 551 (Mar., 2013) A94, [arXiv:1301.3930].

[11] A. A. Abdo, et al., Discovery of High-energy Gamma-ray Emission from the Binary System PSR B1259-63/LS 2883 around Periastron with Fermi, ApJ, 736 (July, 2011) L11, [arXiv:1103.4108].

[12] P. H. T. Tam, et al., Discovery of GeV $\gamma$-ray Emission from PSR B1259-63/LS 2883, ApJ, 736 (July, 2011) L10, [arXiv:1103.3129].

[13] D. Khangulyan, et al., Post-periastron Gamma-Ray Flare from PSR B1259-63/LS 2883 as a Result of Comptonization of the Cold Pulsar Wind, ApJ, 752 (June, 2012) L17, [arXiv:1107.4833].

[14] S. V. Bogovalov, et al., Modelling interaction of relativistic and non-relativistic winds in binary system PSR B1259-63/SS2883 - I. Hydrodynamical limit, MNRAS, 387 (June, 2008) 63-72, [arXiv:0710.1961].

[15] G. Dubus, B. Cerutti, and G. Henri, Relativistic Doppler-boosted emission in gamma-ray binaries, $A \& A, 516$ (June, 2010) A18, [arXiv:1004.0511].

[16] S. W. Kong, K. S. Cheng, and Y. F. Huang, Modeling the Multiwavelength Light Curves of PSR B1259-63/LS 2883. II. The Effects of Anisotropic Pulsar Wind and Doppler Boosting, ApJ, 753 (July, 2012) 127, [arXiv:1205.2147].

[17] G. Dubus and B. Cerutti, What caused the GeV flare of PSR B1259-63?, A\&A, 557 (Sept., 2013) A127, [arXiv:1308.4531].

[18] P. H. T. Tam, et al., High-energy Observations of PSR B1259-63/LS 2883 through the 2014 Periastron Passage: Connecting X-Rays to the GeV Flare, ApJ, 798 (Jan., 2015) L26, [arXiv:1412.2483].

[19] A. Sierpowska and W. Bednarek, $\gamma$-rays from cascades in close massive binaries containing energetic pulsars, MNRAS, 356 (Jan., 2005) 711-726, [astro-ph/0410304].

[20] B. Cerutti, J. Malzac, G. Dubus, and G. Henri, Modeling the three-dimensional pair cascade in binaries. Application to LS 5039, A\&A, 519 (Sept., 2010) A81, [arXiv:1006.2683].

[21] G. Dubus, Gamma-ray absorption in massive X-ray binaries, A\&A, 451 (May, 2006) 9-18, [astro-ph/0509633].

[22] P. Roustazadeh and M. Böttcher, Very High Energy Gamma-ray-induced Pair Cascades in Blazars and Radio Galaxies: Application to NGC 1275, ApJ, 717 (July, 2010) 468-473, [arXiv:1005.2379]. 
[23] P. Roustazadeh and M. Böttcher, Synchrotron Emission from Very High Energy Gamma-Ray-induced Pair Cascades in Active Galactic Nucleus Environments, ApJ, 750 (May, 2012) 26, [arXiv:1202.6084].

[24] I. Sushch and M. Böttcher, Pair cascades in the disk environment of the binary system PSR B1259-63/LS 2883, Journal of High Energy Astrophysics 3 (Sept., 2014) 18-28, [arXiv:1408.6426].

[25] A. T. Okazaki, et al., Hydrodynamic Interaction between the Be Star and the Pulsar in the TeV Binary PSR B1259-63/LS 2883, PASJ, 63 (Aug., 2011) 893-, [arXiv:1105.1481].

[26] J. Takata, et al., Modeling High-energy Light curves of the PSR B1259-63/LS 2883 Binary Based on 3D SPH Simulations, ApJ, $\mathbf{7 5 0}$ (May, 2012) 70, [arXiv:1203.2179].

[27] B. van Soelen, et al., Anisotropic inverse Compton scattering of photons from the circumstellar disc in PSR B1259-63, MNRAS, 426 (Nov., 2012) 3135-3147. 